\title{
Evaluation of Bone Tissue Formation in a Flat Surface Attachment of a Bone Conduction Implant: A Pilot Study in a Sheep Model
}

\author{
Måns Eeg-Olofsson ${ }^{a}$ Agneta Lith $^{c}$ Bo Håkansson ${ }^{d}$ Sabine Reinfeldt ${ }^{d}$ \\ Hamidreza Taghavid $^{d}$ Karl-Johan Fredén Jansson ${ }^{d}$ Carina B. Johansson ${ }^{b}$ \\ a Department of Otolaryngology, Head and Neck Surgery, Sahlgrenska Academy, University \\ of Gothenburg, Departments of ${ }^{b}$ Prosthodontics/Dental Materials Science and ${ }^{\mathrm{C}}$ Oral and \\ Maxillofacial Radiology, Institute of Odontology, Sahlgrenska Academy, University of \\ Gothenburg, and dDepartment of Signals and Systems, Chalmers University of Technology, \\ Gothenburg, Sweden
}

\section{Key Words}

Bone conduction · Implantable bone conduction hearing devices · Bone conduction implant . Osseointegration · Histomorphometry · Cone beam computed tomography

\begin{abstract}
The bone conduction implant (BCI) is a new bone conduction hearing device implanted under intact skin. The transducer has a flat direct contact with the mastoid part of the temporal bone, and no screws are used. The sound signal is transmitted from the external audio processor to the implant by means of magnetic induction. In this study, osseointegration of a flat passive $\mathrm{BCI}$ transducer dummy in sheep skulls was assessed using quantitative and qualitative histology as well as cone beam computed tomography (CBCT) and computed tomography (CT). The histology results were also related to the mechanical properties of the bone-to-implant interface. Eight months after the surgical implantation, histology sections of the bone close to the implant showed bone remodeling, compact bone and osseointegration. The histological findings corresponded well to the mechanical measurements indicating stiffer bone close to the implant and unaffected skull vibration transmission. Neither CBCT nor CT had enough resolution to visualize the bone-to-implant interface in detail. In this study, using an animal model, it is shown that a flat implant in contact with bone can be a feasible method for efficient vibration transmission to the skull bone.




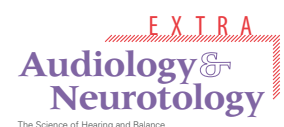

\begin{tabular}{l|l}
\hline \multicolumn{2}{l}{ Audiol Neurotol Extra 2014:4:62-76 } \\
\hline DOI: 10.1159/000369918 & $\begin{array}{l}\text { (c) 2014 S. Karger AG, Basel } \\
\text { www.karger.com/aue }\end{array}$ \\
\hline Eeg-Olofsson et al.: Evaluation of Bone Tissue Formation in a Flat Surface Attachment
\end{tabular} of a Bone Conduction Implant: A Pilot Study in a Sheep Model

\section{Introduction}

\section{Background}

In conventional bone conduction devices the transducer, or actually its casing, is applied with a static force towards the intact skin and underlying soft tissues using a steel spring or the frames of a pair of glasses. This type of hearing devices is sometimes referred to as 'skin drive' bone conduction devices. With the introduction of bone-anchored hearing aids (BAHA), a significant improvement in the treatment of patients with conductive or mixed hearing loss was achieved [Tjellström et al., 1981]. The BAHA transducer is attached to the skull via a skinpenetrating abutment and transmits vibrations directly into the bone, sometimes referred to as 'direct drive' bone conduction. The abutment is in turn attached to a threaded titanium fixture which is inserted, and preferably osseointegrated, in the bone (fig. 1a).

There are side effects for users of BAHA mainly associated with the percutaneous implant solution [de Wolf et al., 2008; Dun et al., 2012; Hobson et al., 2010; Wallberg et al., 2011]. To avoid these complications, daily maintenance is recommended by cleaning the area around the abutment. Although not scientifically assessed, it can also be difficult to attach the BAHA to the abutment, and the esthetics of the percutaneous abutment penetrating the skin is also a debated issue.

Extensive research in the field of bone conduction hearing devices has led to a development towards intact skin solutions. The bone conduction implant (BCI) is a new implantable bone conduction device where the transducer is placed in the temporal bone and covered by intact skin [Eeg-Olofsson et al., 2014]. The BCI consists of an implanted part called the Bridging Bone Conductor and an externally worn audio processor including the digital sound processor, microphones and battery (fig. 1b, c). The BCI transducer casing is placed in a drilled 4- to 5 -mm-deep recess in the mastoid part of the temporal bone approximately $10-15 \mathrm{~mm}$ from the posterior edge of the bony ear canal opening and is secured in its position by a slight pressure to the bone. Sound signals are transferred through the intact skin and soft tissues by magnetic induction and are then further transmitted to the implanted BCI transducer where the signals are converted into mechanical vibrations. The BCI transducer has direct contact with the underlying bone, and thus sound transmission to the cochlea is not attenuated by the skin and subcutaneous tissues. A screw attachment in the mastoid region is not an optimum solution because (1) there are frequent air cells that might affect a screw attachment negatively, especially in the medial portion of a recess where the transducer preferably should be attached for obtaining maximal stimulation of the cochlea, (2) it should be easy to install and, if needed, to replace the implant, and (3) the size of the implant should be as small as possible.

The BCI transducer casing has a flat contact with the bone in the bottom plane of the drilled mastoid recess. A static pressure is needed between the transducer casing and the bone in order to transmit vibrations. This static pressure is provided by a thin titanium wire, which is inserted through small drilled holes in the bone bed on both sides of the BCI transducer and then tightened by the surgeon. The interface between the bone and the transducer casing is important in the BCI since possible soft tissue formation or severe bone resorption over time might have a detrimental effect on sound transmission to the cochlea. Therefore, this new method with flat surface vibration transmission from the transducer casing to the bone raises a number of research questions: will the vibration transmission be stable over time? Will the contact force be maintained over time or will any nonlinear signal transmission arise? Will soft tissue form and bone resorb in the contact area between the implant and the bone or, instead, will bone remodel in the surrounding bone bed?

Osseointegration was described by Brånemark et al. [1977]. The term osseointegration was defined in 1981 by Albrektsson et al. [1981] and in 1985 by Brånemark [1985]. However, there is no consensus about the percentage of bone-implant contact (BIC) needed to be clas- 


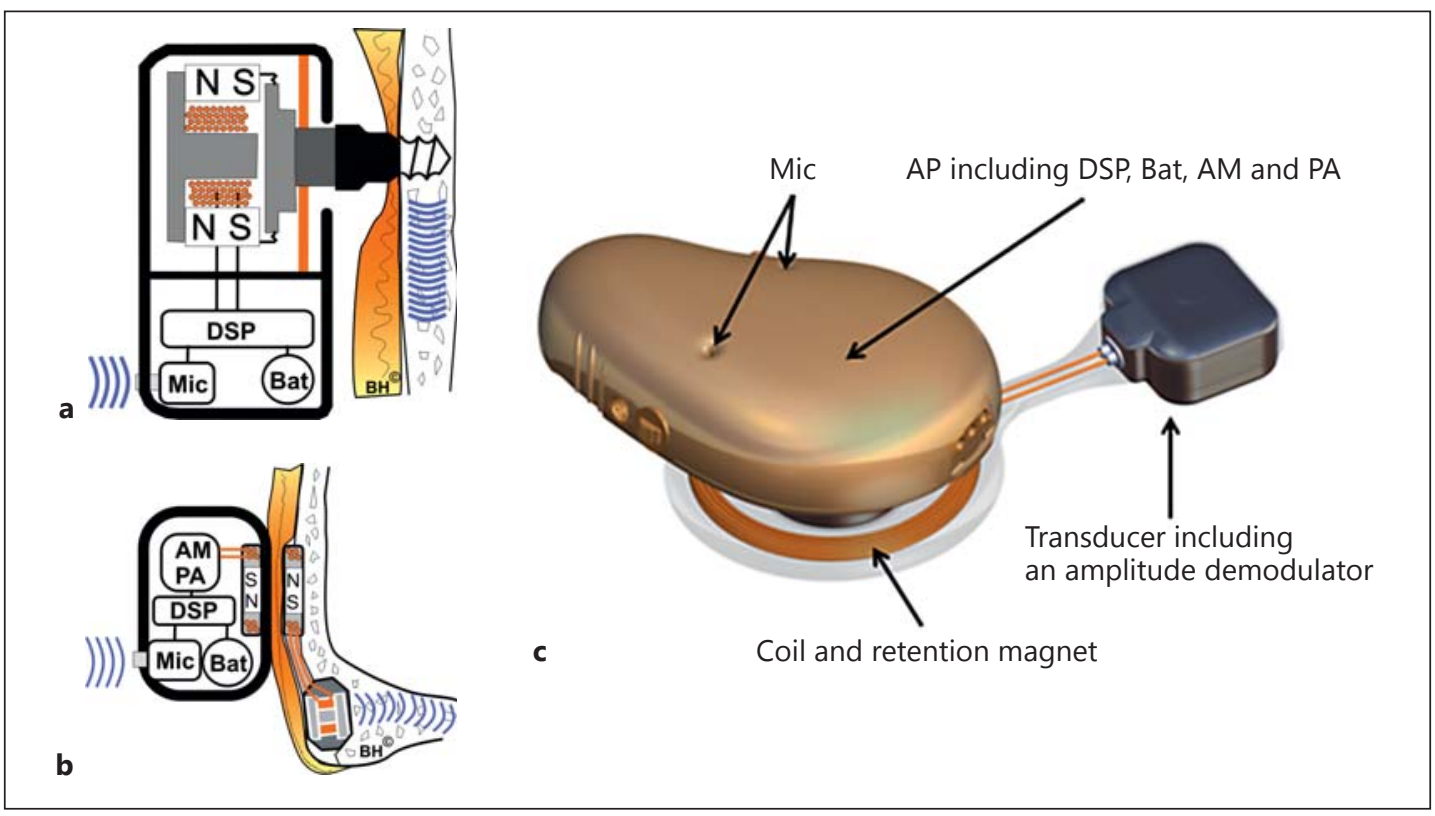

Fig. 1. a The principal design of the bone-anchored hearing aid comprising a bone-anchored fixture and skinpenetrating abutment to which an external sound processor is attached. The sound processor also includes the transducer. $\mathbf{b}$ The principal design of the BCI comprising an implanted unit, including the transducer, and an external audio processor. $\mathbf{c}$ The $\mathrm{BCI}$ system. $\mathrm{AM}=$ Amplitude modulator; $\mathrm{AP}=$ audio processor; $\mathrm{Bat}=\mathrm{bat}-$ tery; DSP = digital sound processor; $\mathrm{Mic}=$ microphone; $\mathrm{N}=$ magnet north pole; $\mathrm{PA}=$ power $\operatorname{amplifier} ; \mathrm{S}=$ magnet south pole.

sified as osseointegrated. Histomorphometrical results from retrieved clinical oral implants being loaded for up to 5 years as well as a group of unloaded implants revealed that there is never a 100\% BIC [Bolind et al., 2005]. Interestingly, and for the first time, it was shown that the osseointegration pattern changed around the implant geometry as well as with the time of loading. A situation with $100 \%$ bone contact would be abnormal since healthy bone is constantly remodeling.

Noninvasive methods using various 3-dimensional (3D) computer tomography methods, such as cone beam computed tomography (CBCT), are important tools in the diagnostic process, treatment planning and outcome assessments of diseases in the craniofacial and dental area. CBCT is regarded as a valuable tool to visualize bone density and air [Adibi et al., 2012; De Vos et al., 2009]; however, to evaluate the contact between bone and screw implants, CBCT is not regarded as sufficient [Buser et al., 2013; Hasan et al., 2014]. In this study, CBCT and computed tomography (CT) were included in the study methods to visualize the interface of flat titanium implants and bone in sheep skulls.

Besides histological and radiological methods to evaluate implant attachment to the bone, there are also objective vibrational measuring methods. By mechanically stimulating the implant, bony structure resistance to vibration velocity can be measured when an exciting force is applied. This resistance is referred to as the mechanical point impedance (MPI), and it provides information about the bone-to-implant stiffness at the specific implant position [Håkansson et al., 1986]. Moreover, the transfer function (TF) measures how a known vibration excitation force in one point of the skull is transmitted to another point of the skull. With soft tissues in the bone-to-implant interface, or lack of bone contact, both MPI and TF are expected to be affected. Typically, they will be lower, but also resonances can move/shift in frequency compared to an interface with a high BIC. 
Preclinical mechanical measurements of MPI and TF on human cadavers [Håkansson et al., 2010] have proven that a flat implant to bone contact is an effective method to transmit sounds to the cochlea. The next phase was to study the mentioned research questions in an animal model (sheep). A first report of this animal study regarding the vibration transmission and linearity has been published [Taghavi et al., 2013]. It was found that the objectively measured TF was stable and linear over time (8 months) and that the MPI increased.

In the current study, the same sheep as in the study of Taghavi et al. [2013] were used.

\section{Aims of Study}

The aim of this study was to describe the bone-to-implant interface in temporal bones extracted from sheep skulls. In more detail the aims are (1) to examine sections through implants in the bone using qualitative and quantitative histology, i.e. histomorphometry, and to evaluate the bone tissue in close vicinity to the bone-to-implant interface; (2) to examine $3 \mathrm{D}$ radiological images of the implant in the bone and to investigate whether the bone-toimplant interface can be observed on the 3D material, and (3) to compare the histomorphometrical data in this study with the results from the previous investigation of the MPI and the transfer response function by Taghavi et al. [2013].

\section{Materials and Methods}

This study was approved by the Animal Ethics Committee in Gothenburg, Sweden.

Three female sheep at the approximate age of 3 years were used in this study. Surgery and implant installation with subsequent mechanical measurements were done according to Taghavi et al. [2013] at time 0 (baseline). Eight months after baseline, the implants were exposed to the same set of mechanical measurements as at baseline. After finishing the measurements, the sheep were sacrificed, and CBCT and CT scans were immediately done on each head followed by extraction of the temporal bones including the implants on both sides of the skull. The temporal bones were immersed in fixative and further processed in the laboratories (see section Histology and Histomorphometry below).

\section{Implant Description}

The dummy implants were solid grade 2 circular titanium cylinders, $6 \mathrm{~mm}$ in diameter and with a height of $2 \mathrm{~mm}$ (fig. 2a, b). The surface topography towards the bone, measured with interferometry, had a roughness of $0.5 \mu \mathrm{m}$ (Sa value) which is considered to be a minimally rough implant surface [Wennerberg and Albrektsson, 2010]. To investigate bone ingrowth, an inscription of 'BCI' was made in the bottom plane in half of the study implants (fig. 2a). The width of the BCI inscription was $250 \mu \mathrm{m}$ and the depth was $40 \mu \mathrm{m}$. During the 8 months between the surgeries, the implants were passive, i.e. no transducer was integrated in the implants.

\section{Surgery}

Surgery of the 3 sheep was made at baseline and after 8 months when the study was terminated. All surgeries and measurement procedures were done under general anesthesia.

Baseline Surgery

A postauricular incision was made down to the mastoid bone approximately $5 \mathrm{~mm}$ behind the ear canal opening. A circular recess of $6 \mathrm{~mm}$ in diameter was drilled in the bone down to clearly visible mastoid air cells. The titanium implant was positioned in flat contact with the bone. One implant was positioned on each side of the skull, and for one implant of each sheep, 


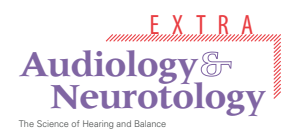

\begin{tabular}{l|l}
\hline \multicolumn{2}{l}{ Audiol Neurotol Extra 2014;4:62-76 } \\
\hline DOI: 10.1159/000369918 & $\begin{array}{l}\text { (C) 2014 S. Karger AG, Basel } \\
\text { www.karger.com/aue }\end{array}$ \\
\hline Eeg-Olofsson et al.: Evaluation of Bone Tissue Formation in a Flat Surface Attachment
\end{tabular} of a Bone Conduction Implant: A Pilot Study in a Sheep Model
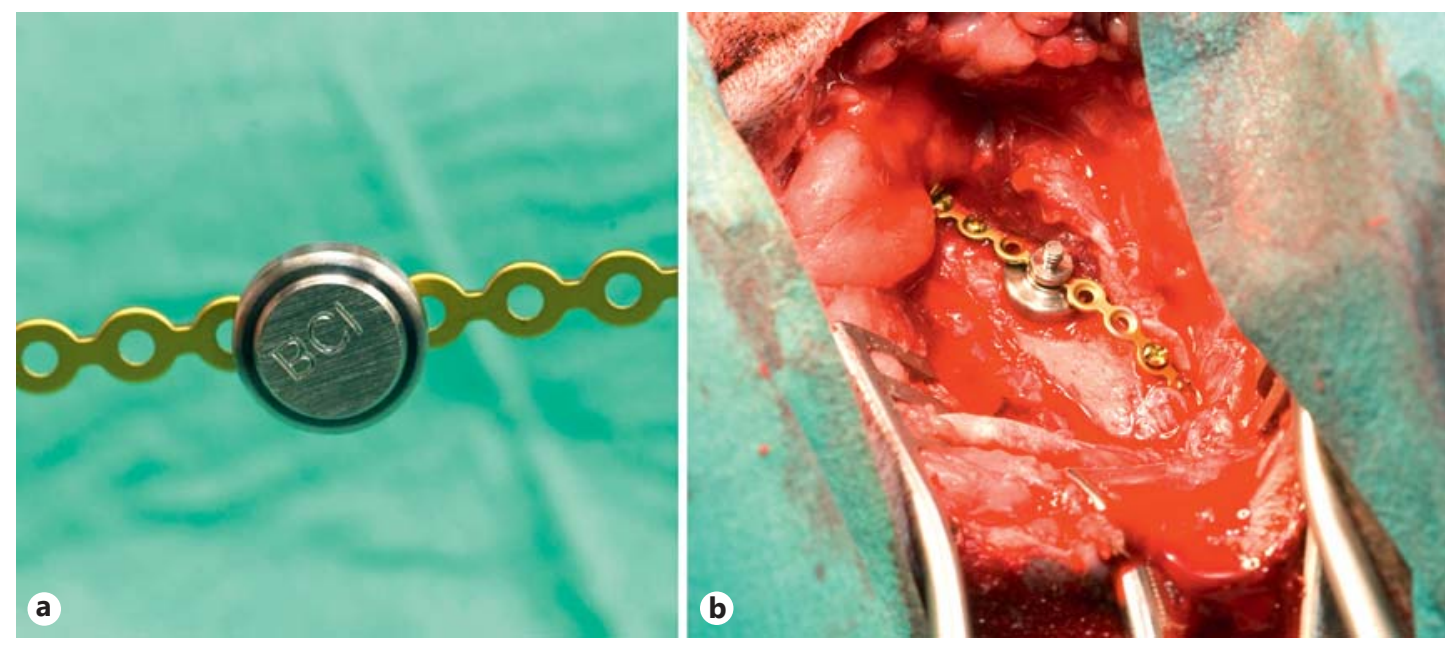

Fig. 2. a The titanium cylindrical implant used in this study; the surface in contact with the bone has the same size as the transducer casing used in the BCI system. b A seat was drilled on both sides of the sheep skull just behind the bony ear canals. A retention plate system with miniature orthopedic screws was used to attach the implant to the skull bone.

a thin layer of bone pâté (remnants of bone particles after drilling in the bone) was smeared between the implant and the mastoid air cells. For the purpose of stabilization and to provide a static pressure towards the bone similar to the BCI transducer, the implants were secured by a 0.6 -mm-thick titanium plate with two $1.2 \times 3 \mathrm{~mm}$ titanium screws (Stryker Corp., Kalamazoo, Mich., USA) on each side of the implant (fig. 2b). A plastic cover screw was attached on top of the implant to minimize trauma from the central fixation screw for the measuring device [Taghavi et al., 2013]. Finally the incision was closed.

Follow-Up Surgery

A postauricular incision was made down to the implant exposing it enough to carry out measurements [Taghavi et al., 2013]. The temporal bones were removed using a Schuknecht bone saw.

\section{Radiological Examination}

CBCT and CT were performed on the sheep skulls. The skulls were placed with the region of the titanium implant in the center of the CBCT indicated by the CBCT light lines. CT axial scans were performed of the whole sheep skulls positioned on their neck.

The CBCT unit consisted of a 3D Accuitomo 170 (J. Morita Mfg. Corp., Kyoto, Japan) with a flat-panel digital detector. The field of view was $100 \times 150 \mathrm{~mm}$ with an isotropic voxel of $0.125 \mathrm{~mm}^{3}$. The exposure parameters were $90 \mathrm{kV}$ and $8 \mathrm{~mA}$ for $17.5 \mathrm{~s}$ as the radiation cone rotated $360^{\circ}$ around the object, resulting in 556 projections. The examination data were primarily reconstructed to contiguous $0.5-\mathrm{mm}$ axial slices in an Accuitomo workstation with the i-Dixel 3D software (J. Morita Mfg. Corp.) and then archived in Sectra IDS5 PACS and further reformatted with multiplanar reformations in Sectra IDS5 PACS MPR (Sectra Imtec, Linköping, Sweden).

The CT unit consisted of a 4-channel multi-slice General Electric LightSpeed QX/i CT scanner (General Electric Medical Systems, Milwaukee, Wis., USA). 302 axial scans were performed with a slice thickness of $1.25 \mathrm{~mm}$ and a slice distance of $1.25 \mathrm{~mm}$. The exposure parameters were $120 \mathrm{kV}, 80 \mathrm{~mA}$ and $1.6 \mathrm{~s} /$ rotation. The axial slices were archived and reformatted with multiplanar reformations in IDS5 PACS MPR (Sectra Imtec). 


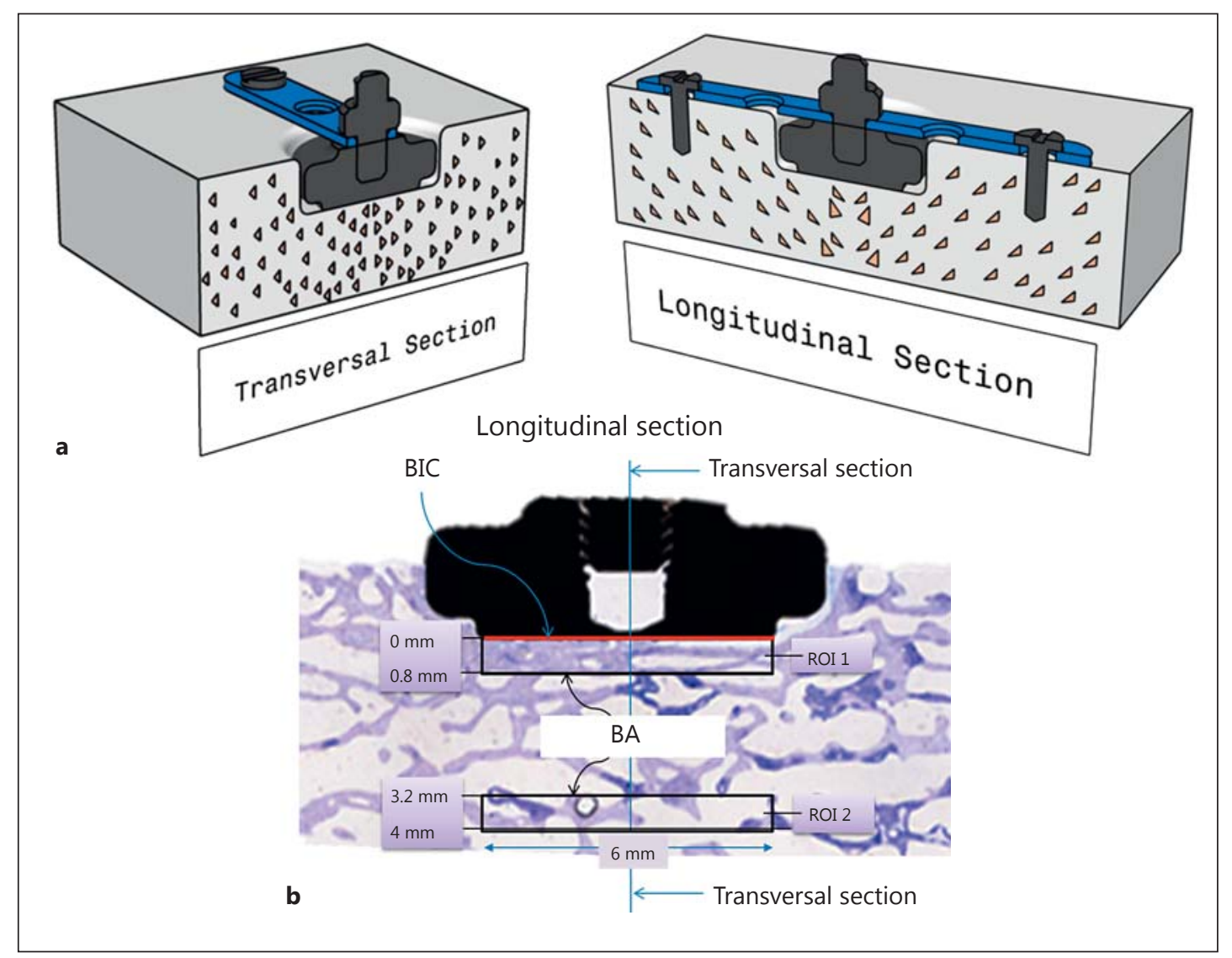

Fig. 3. a Two sections were prepared in longitudinal direction through the retention plate and screws, and one section was taken in transversal direction $\left(90^{\circ}\right.$ angulation to the longitudinal cut). b The histomorphometrical quantifications involved the percentage of BIC in the interface and the percentage of BA measured in standardized regions denoted as ROI. In this study one ROI was just below the implant (ROI 1: $6.0 \times 0.8$ $\mathrm{mm}$ at 0-0.8 $\mathrm{mm}$ depth) and one was more medial for comparison (ROI 2: $6.0 \times 0.8 \mathrm{~mm}$ at 3.2-4.0 $\mathrm{mm}$ depth).

\section{Histology and Histomorphometry}

The retrieved temporal bone samples with surrounding bone tissue were immersed in $4 \%$ neutral buffered formaldehyde fixation for 2 weeks followed by rinsing in tap water and dehydration in a series of ethanol, from $70 \%$ to absolute ethanol. First, resin infiltration was conducted in diluted resin, followed by infiltration in pure resin under stirring and vacuum conditions. The final step involved embedment in pure resin (Technovit 7200 VLC, Kulzer, Germany) and curing in UV light.

Nondecalcified cut and ground sections of a thickness of $15 \mu \mathrm{m}$ were prepared using the Exakt ${ }^{\circledR}$ equipment (EXAKT Technologies Inc., Oklahoma City, Okla., USA) and the technique ad modum Donath [Donath and Breuner, 1982; Johansson and Morberg, 1995]. In brief, the cured resin-embedded samples were first divided in a longitudinal manner aligned with the titanium plate and the screws. Two sections were taken for the longitudinal cuts. The two remaining blocks were glued together and then divided by a transversal cut. One section was prepared from this cut (fig. 3a). The sections were histologically stained in toluidine blue mixed in pyronin G. Altogether three sections from each sample were quantitatively and qualitatively analyzed using a semiautomatic image analysis program Microvid ${ }^{\mathrm{TM}}$ coupled to a Leitz Aristoplan light microscope (Leitz, Wetzlar GmbH, Germany). 
BIC is a 1-dimensional measure of the bone-to-implant contact in percent along a specified interface line of a certain length, in this study as long as the implant diameter, i.e. $6.0 \mathrm{~mm}$. The bone area (BA) is a 2-dimensional measure with the same interface length, where the percentage of bone is specified within an area below the implant, preferably one area immediately below the implant and one area more medially for comparison. Such areas are also referred to as regions of interest (ROIs). Histomorphometry, using BIC and BA, is a method to quantify the bone tissue adjacent to implants, where BIC is an indicator of the percentage of the surface that is osseointegrated. Both BIC and BA were calculated as an average from the two longitudinal sections and one transversal section. BIC was measured along the interface at the flat implant surface facing the bone tissue. BA measurements were performed in two ROIs, one close to the surface of the implant (ROI 1: 0-0.8 mm depth) and one more medially under the implant surface (ROI 2: 3.2-4.0 mm depth) (fig. 3b). Each ROI was as long as the implant surface diameter $(6.0 \mathrm{~mm})$ facing the bone tissue. For the transversal cut, the length of each ROI was slightly shorter due to the previously obtained section for the longitudinal cut. A ROI thickness of $0.8 \mathrm{~mm}$ was found suitable with regard to the size of the implant.

\section{Results}

Sheep No. 3 suffered from a wound infection on the left side. Antibiotic treatment did not cure the infection, and the sheep was sacrificed after 4 months. Mechanical measurements therefore had to be limited to MPI on the right (noninfected) side. CBCT and CT scans as well as histological preparations were done after termination. BIC and BA were not calculated on the infected side of sheep No. 3 due to the non-bone integrated surface with a thick soft tissue formation between the implant and the bone. Sheep No. 1 and 2 completed the time to the second surgery with subsequent mechanical measurements, CBCT and CT scans and histological preparations. The implants in sheep No. 1 were abandoned before removal of the temporal bones since the implants were clinically unstable. Reasons for this instability were assumed to be repeated 'trauma' behind the ears using its back leg hooves as well as using fence poles. The implants were therefore subjected to external trauma in spite of the fact that they were situated underneath the skin. Thus, no implant from sheep No. 1 was part of the histological examination, and therefore no BIC or BA calculations were possible on sheep No. 1. From a clinical point of view, the most important results were obtained from sheep No. 2 since these implants were firmly attached at 8 months after baseline, and therefore only results from sheep No. 2 are described as follows. Results from the other sheep will be included in the discussion.

\section{CBCT and CT}

A reformatted CBCT image (fig. 4a) as well as a reformatted CT image (fig. 4b) of the titanium implants show bone tissue embedding the implants. In both techniques a layer of more dense bone is noted just below the bone-to-implant interface. The implants, screws and plates are in contact with bone although the inferior part of the plate is just outside the bone on the left side.

\section{Qualitative Observations on the Samples of the Selected Sheep No. 2}

For the sample with bone pâté added underneath the implant at the time of surgery, the implant was partly integrated in the spongeous bone (fig. 5a-c). In the soft tissue regions close to the implant, inflammatory cells (macrophages) could be observed, but in general they were sparse. A bone collar was formed under the device and most of the screws securing the plate were integrated in bone. However, one of the screws was not osseointegrated, and a channel of unknown origin ended in the screw cavity (fig. 5a, second screw from the right). 

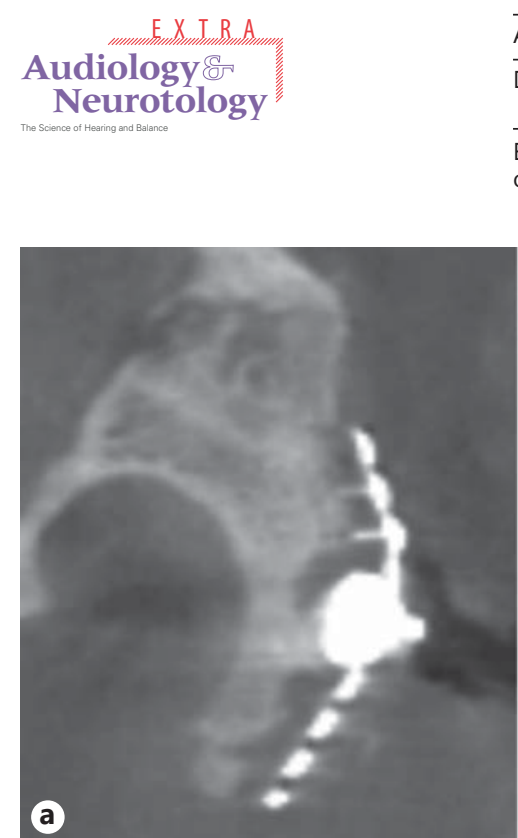

Audiol Neurotol Extra 2014;4:62-76

DOI: $10.1159 / 000369918$

Eeg-Olofsson et al.: Evaluation of Bone Tissue Formation in a Flat Surface Attachment of a Bone Conduction Implant: A Pilot Study in a Sheep Model

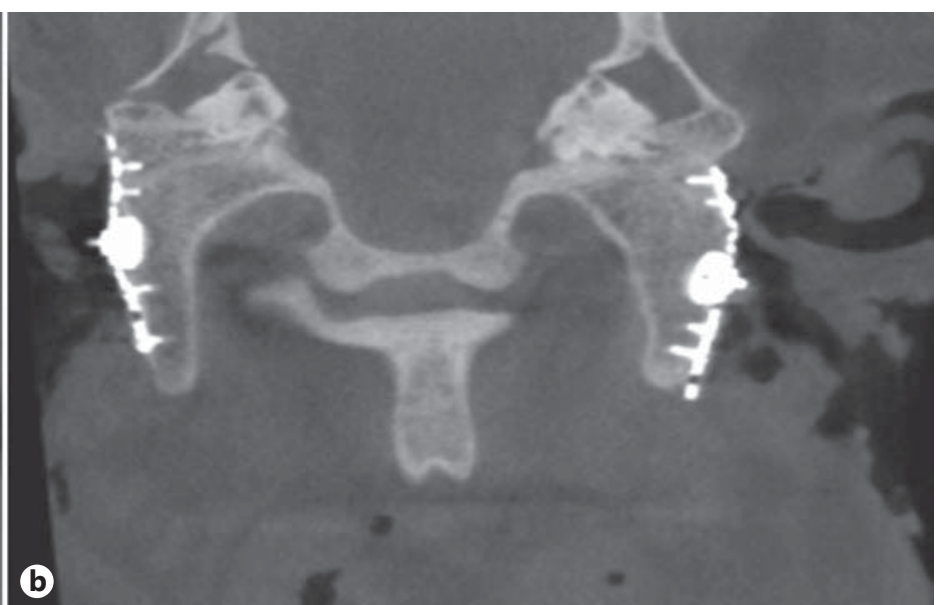

Fig. 4. CBCT (a) and CT (b) images of implants, retention plates and screws on both sides of sheep No. 2.

At higher magnification, newly formed bone in contact with the implant surface was noted. Old bone particles undergoing resorption could be seen, and quite large osteoclasts were frequently noted on these bone surface regions although they were not observed in the interface region. Moreover, osteoclasts were observed in a front area of a cylinder-shaped formation inside the bone, and osteoblasts were observed on the osteoid rims behind the osteoclast resorption front. This observation is indicative of internal bone remodeling, i.e. similar to a cutting cone (fig. $5 \mathrm{c}$ ).

For the sample without bone pâté (fig. $5 \mathrm{~d}-\mathrm{f}$ ), a similar survey picture was demonstrated showing mostly mature bone with an ongoing bone remodeling activity and no major foreign body reaction. The soft tissue around the implant sides and under the metal plate was less pronounced compared to the other side, i.e. this implant demonstrated a greater integration.

\section{$B I C$ and $B A$}

No statistical analyses were performed due to small sample size. All objective results regarding BIC and BA are presented in figure 6. After 8 months of follow-up, histomorphometrical data (BIC and BA) demonstrated average values of $23 \%$ BIC, $69 \%$ BA in ROI 1 and $29 \%$ BA in ROI 2 for the implant with bone pâté added. The corresponding mean values for the other implant, without bone pâté added, were 28\% BIC, 69\% BA in ROI 1 and 32\% BA in ROI 2. Thus, no obvious difference was noted when comparing histomorphometrical results of using bone pâté or not using bone pâté in the interface. By comparing BA at ROI 1 and ROI 2, approximately $40 \%$ more bone is found near the implant surface compared to an area slightly below.

Fig. 5. Sheep No. 2, left side, with bone pâté added at baseline surgery: longitudinal cut (a) and transversal cut (b). c Bone tissue is partly filling the BCI inscription. Bone forming cavities can be seen close to the implant surface. The 'canal-like structure' in the center indicates ongoing internal remodeling of bone, i.e. a cutting cone-like structure with osteoclasts in the front and osteoid rims with osteoblast behind. Sheep No. 2, right side, without bone paté: longitudinal cut (d) and transversal cut (e). $\mathbf{f}$ Newly formed bone as well as an ongoing bone formation can be observed close to the implant surface whereas the center part demonstrates more compact bone. In some regions, bone remodeling cavities (lighter appearance) can be seen. All four sections $(\mathbf{a}, \mathbf{b}, \mathbf{d}, \mathbf{e})$ demonstrate bone condensation in close vicinity to the implant surface.

(For figure see next page.) 
Audiology
Neurotology

\begin{tabular}{l|l}
\hline \multicolumn{2}{l}{ Audiol Neurotol Extra 2014;4:62-76 } \\
\hline DOI: 10.1159/000369918 & $\begin{array}{l}\text { @ 2014 S. Karger AG, Basel } \\
\text { www.karger.com/aue }\end{array}$ \\
\hline
\end{tabular}

Eeg-Olofsson et al.: Evaluation of Bone Tissue Formation in a Flat Surface Attachment of a Bone Conduction Implant: A Pilot Study in a Sheep Model
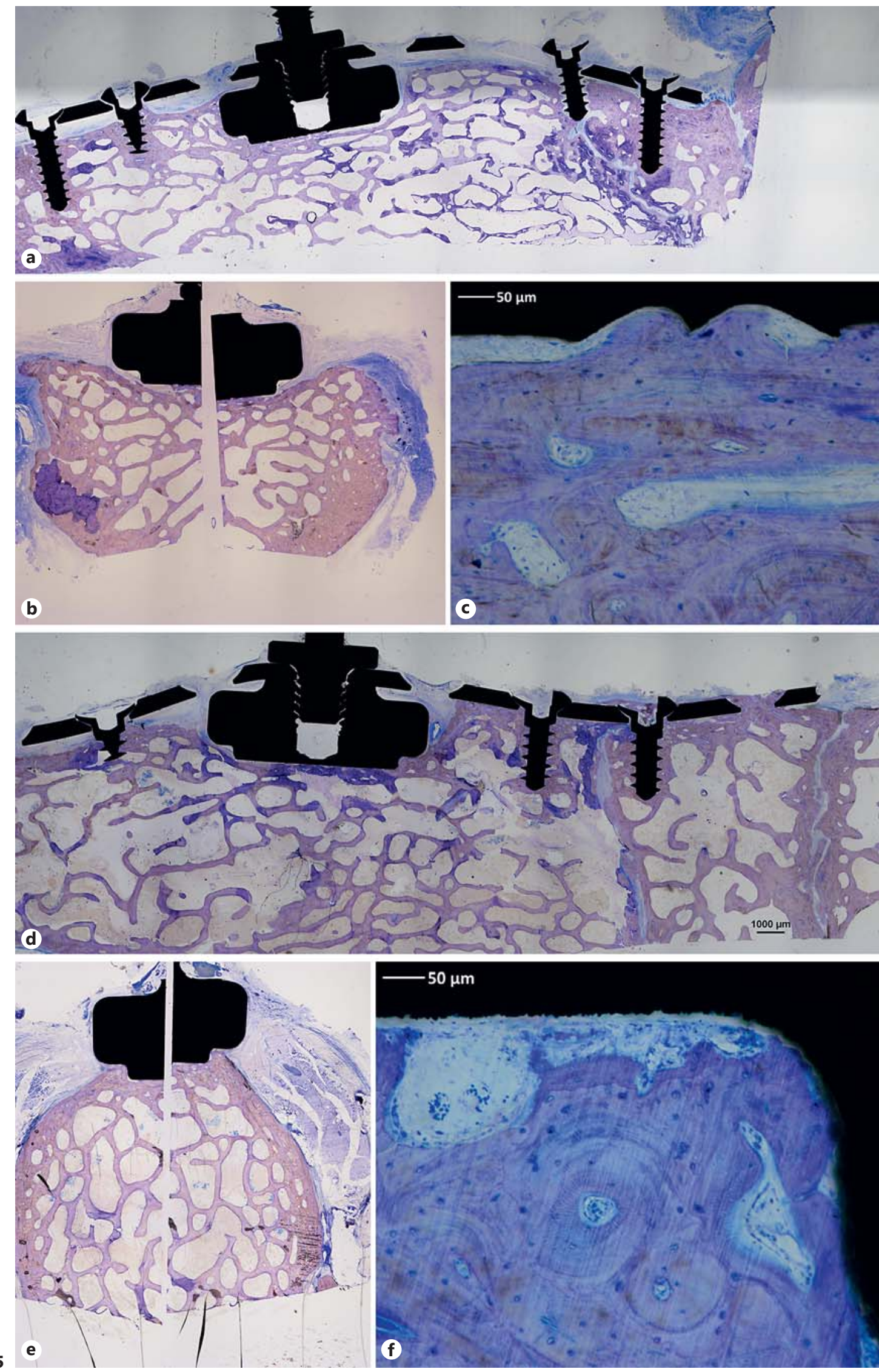

KARGER 


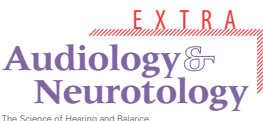

Fig. 6. The average BIC and BA values for two implants in sheep No. 2, where one side was prepared with bone pâté in the interface at installation, whereas no bone pâté was added on the other side. The BIC and BA averages are calculated from three histological sections $(n=3)$. Bars indicate the maximum and minimum range of BIC and BA.

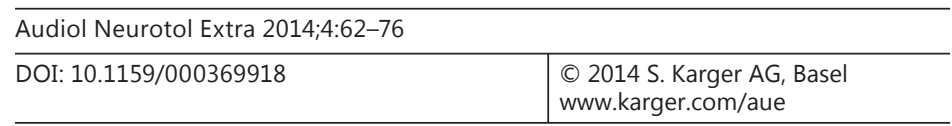

Eeg-Olofsson et al.: Evaluation of Bone Tissue Formation in a Flat Surface Attachment of a Bone Conduction Implant: A Pilot Study in a Sheep Model

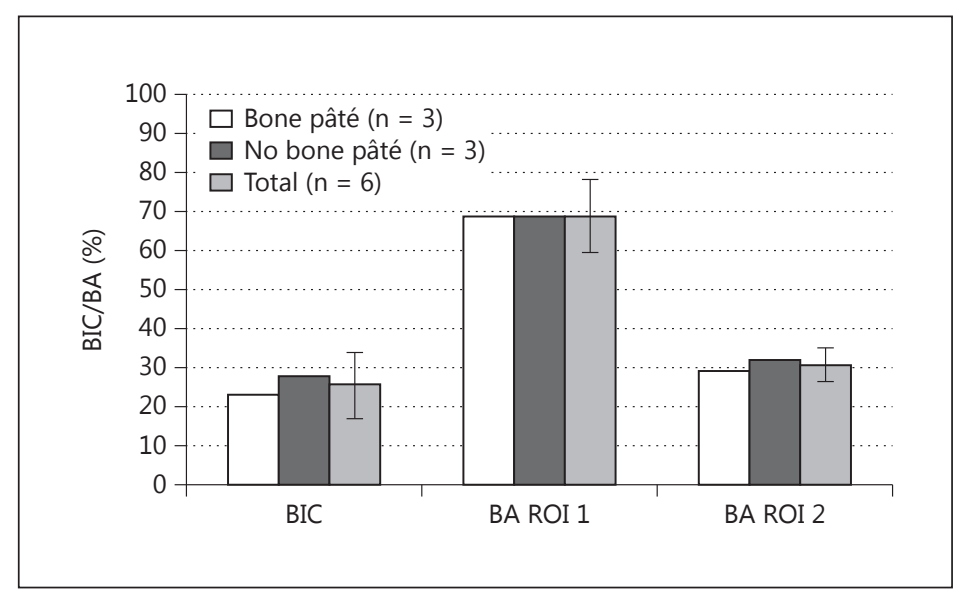

\section{Discussion}

One of three sheep completed the study as intended in the study protocol. The small sample size is a weakness from a scientific point of view. However, the authors do believe that the results of the study can contribute to increased understanding of the interaction between bone and titanium implants.

For practical reasons, it was not possible to cut the temporal bone samples so that the whole area of the bone-to-implant interface could be examined. The longitudinal cuts were positioned to include the screws holding the plate. The transversal cut added information about the bone-to-implant interface for the area perpendicular to the two longitudinal cuts. In this way, a good approximation of the bone-to-implant interface of the whole area was achieved from three cuts.

Sheep skulls were in this study from an anatomical point of view deemed to be a good model to draw some conclusions for the live human. On the other hand, sheep seem to be sensitive to wounds and surgical interventions. An important reason for the implants in sheep No. 1 to be loose and also for the implant in the noninfected side (right) in sheep No. 3 to be loose was back leg kicking in the back-to-front direction. If using sheep as a model, it is recommended that surgical interventions are made at locations where the sheep cannot reach with its hooves. The loose implants in sheep No. 1 were extracted before histomorphometry, first since they were not judged to stay in place when the temporal bones were removed and second because it was an opportunity to examine the bony surface beneath the implant macroscopically. The loose and moving implants resulted in a bowl-shaped and compact bony surface of the recess for the implants. The histological cuts also revealed soft tissues between the implant and the bone (fig. 7).

One detail which is not understood are the channels filled with soft tissue that open into the implant recesses. The presence of these channels seems to be correlated to the amount of soft tissue around the implant. This correlation is clear for both sides in sheep No. 1 (fig. 7, 8) and the right side in sheep No. 3. On the left side, in sheep No. 2 (fig. 5a), such a channel opens into a screw in its position, and the lack of bone around this screw is clear. It is not known whether these channels are a result of the implantation or whether they are a normal anatomical feature in the sheep skull, for example a cranial suture. Soft tissue channels anatomically situated in a recess could imply a risk of soft tissue invasion around the implant and could result in a suboptimal efficiency of the device. 


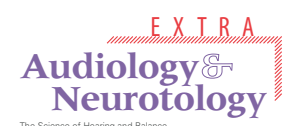

Audiology
Neurotology

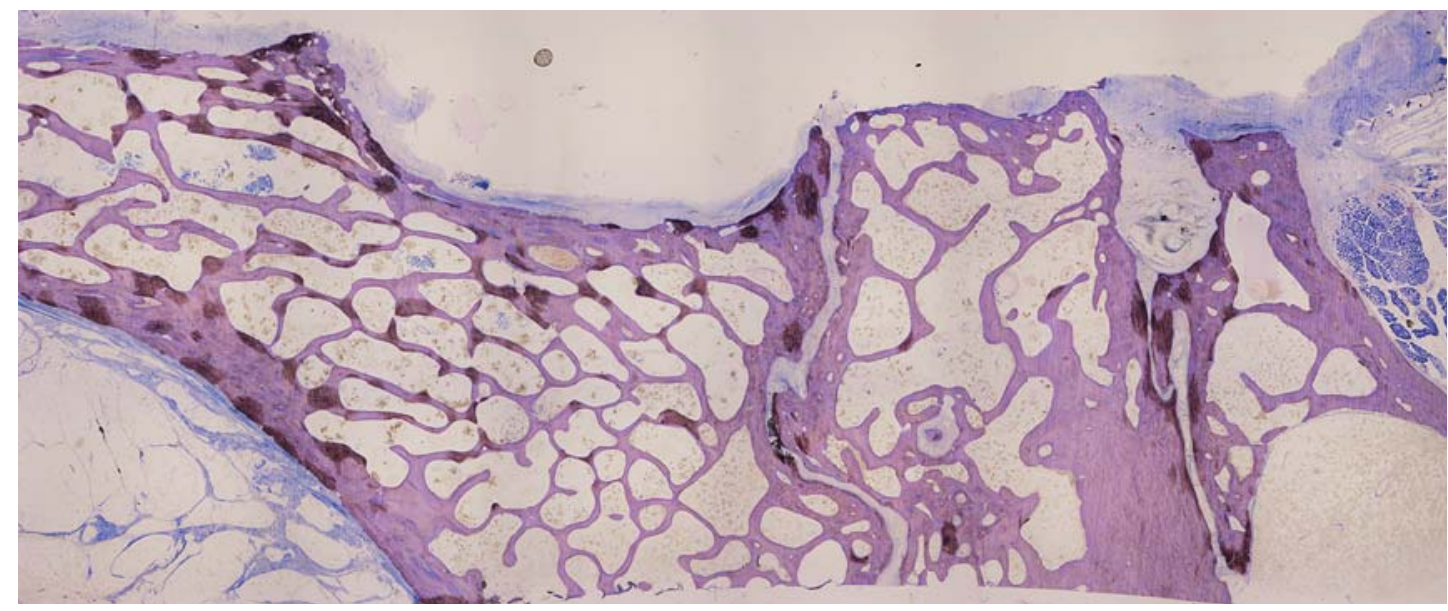

Fig. 7. Histological section from the longitudinal cut of sheep No. 1, left side.

\section{Qualitative and Quantitative Observations Compared with Mechanical Measurements}

In the same sheep as in the current study, MPI and TF measurements were performed [Taghavi et al., 2013]. The BCI transducer has a flat direct contact with the underlying bone, and over time the bone in contact with an implant like the BCI is expected to remodel and the implant to be osseointegrated. Osseointegration will likely increase the MPI, but TF will remain unchanged. A decrease in the MPI and the TF would probably imply worse vibration transmission and therefore less sound stimulation of the cochlea. On both sides in sheep No. 2 , with a mean value of $25 \%$ BIC and a mean value of $70 \%$ BA close to the implant surface, MPI increases from baseline to the measurements 8 months after baseline, and TF remains unchanged. This finding indicates that the contact between the implant and the bone strengthens over time.

Although observing soft tissue formation partially beneath the implant on the left side in sheep No. 2 (fig. 5a), the MPI increases and the TF remains unchanged. It is also evident that in the case of soft tissue growth between the implant and the bone without direct contact as in sheep No. 1, both MPI and TF decrease. It has been shown in cadavers [Håkansson et al., 2010] that a limited bone-to-implant contact is sufficient to transfer sound to the inner ear efficiently. The results in the current animal study confirm the findings by Håkansson et al. [2010]. In a live human, it means that even if the implant has a limited contact with the bone, as expected in a bony structure like the air cells in the mastoid bone, the stimulation to the inner ear will be sufficient. By filling cavities in the mastoid bone with bone pâté, the hypothesis was that BIC and MPI would be greater 8 months after baseline. Neither in our results nor in those by Taghavi et al. [2013], was there any evidence or indication that bone pâté had such an effect.

On one implant per sheep, on the implant surface facing the bone, a $40-\mu \mathrm{m}$-deep inscription of BCI was blasted. At high magnification of the bone-to-implant interface of sheep No. 2 (fig. 5c), the bone formation is ongoing in the groove, and bone forming regions can be observed as lighter stained rims. Large bone remodeling regions are also visible. This is clear evidence that the implant has been stable and that the bone can fill irregularities in the boneto-implant interface making it even more stable. In figure $5 f$, it is also seen that bone is filling out the step from the centermost implant surface to the more peripheral implant surface facing the bone. The bone tissue in close relation to the implant surface is newly formed. Some areas demonstrate ongoing bone remodeling. In case of having to remove the implant, grooves 

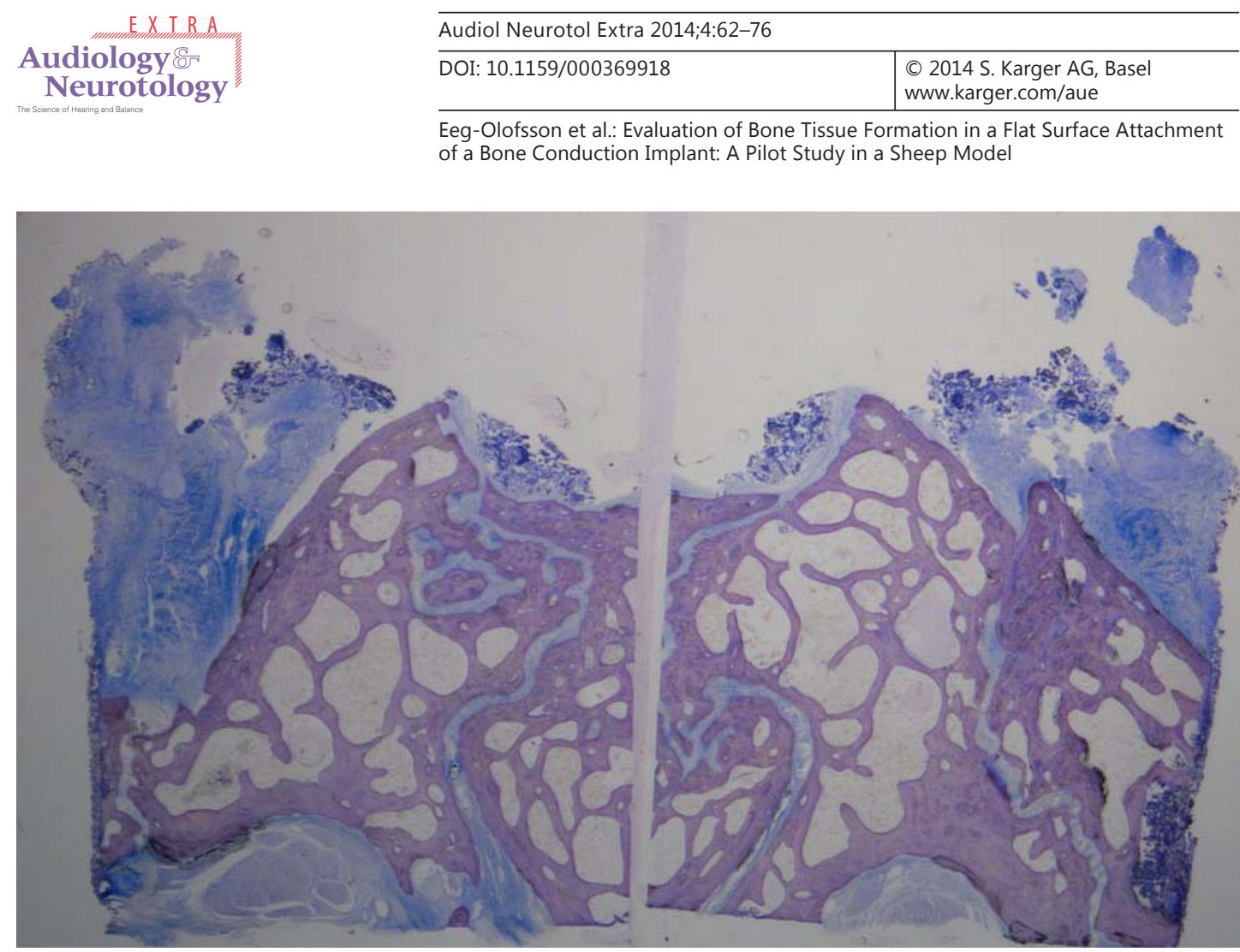

Fig. 8. Histological section from the transversal cut of sheep No. 1, right side.

or irregularities of the implant surface can imply that a higher force is needed to pull out the implant [Rønold and Ellingsen, 2002]. There is a continuous discussion when using screwshaped implants intraorally or extraorally about what the most optimal roughness of the implant surface should be to promote osseointegration. In the case of a flat surface implant like the $\mathrm{BCI}$, this discussion may not be of great interest for an effective and long-lasting transmission of sound to the cochlea.

\section{CBCT and CT}

CBCT and CT were included in the methods since they are noninvasive possibilities to assess the interface between implant and bone. Especially CBCT is important due to its generally lower radiation dose compared to CT [Adibi et al., 2012]. To the best of the authors' knowledge, there are no reported studies on either CBCT or CT on flat hearing implants, where the transducer is in contact with bone. A recent human cadaver study [Guldner et al., 2013] concluded that a similar hearing implant, the Bonebridge ${ }^{\mathrm{TM}}$ (Med-El, Innsbruck, Austria), and surrounding anatomical structures could be visualized using CT and CBCT, where CBCT had an advantage in image quality. The flat medial surface of the Bonebridge transducer is, however, not in contact with bone.

In figure 9, the implant of the infected left side of sheep No. 3 is illustrated with CBCT (fig. 9a) and with CT (fig. 9b). In these images, signs of infection and associated bone resorption are clearly seen. In the CBCT, bone seems to have partly contact with the implant, which is not seen in the CT. The difference is due to a metal artifact in the CBCT image. In figure 5a, part of the implant of the left side of sheep No. 2 is not in contact with bone, and one of the screws is not osseointegrated. This more detailed information, however, cannot be visualized with CBCT or CT (fig. $4 \mathrm{a}, \mathrm{b}$ ). In case of malfunction of the implant, or complications related to the implant, both CBCT and CT could be valuable tools for treatment guidance and possibly also for better understanding of bone remodeling around the implant, but due to the relatively low resolution no information corresponding to BIC nor BA can be obtained using CBCT or CT. In 


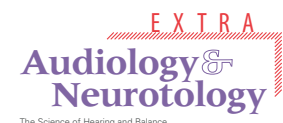

Audiology
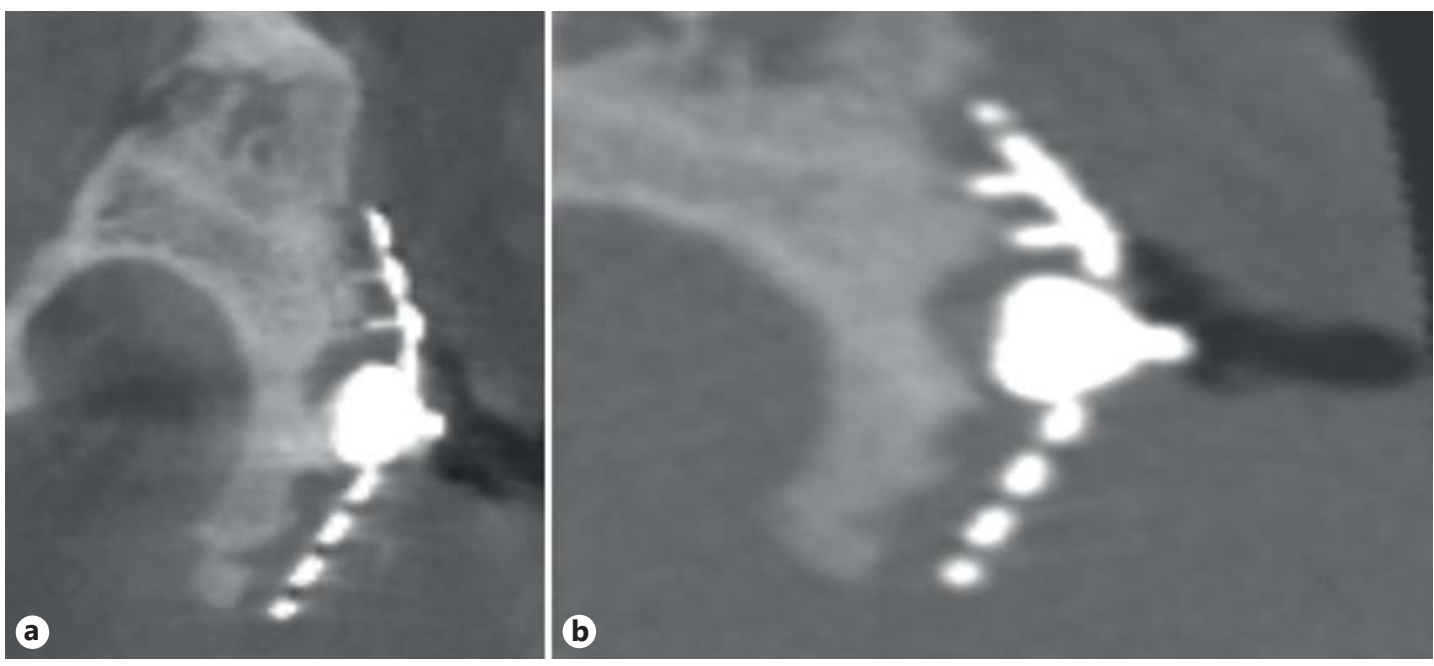

Fig. 9. СВСТ (a) and CT (b) of the implant, screws and plate of the left side of sheep No. 3.

the current study, there was no advantage or disadvantage in this respect regarding the two methods (CBCT and CT). The dense bone just beneath the implant can imply an inflammatory reaction when interpreting $\mathrm{CBCT}$ and CT images. However, we know from the histological cuts in this study that there is a collar of more dense bone close to the bone-to-implant interface indicating that a stiffer bone structure is developed over time. This fact is also shown by the higher MPI results.

\section{Bone Conduction Hearing Devices and Osseointegration}

For bone conduction hearing devices using a screw attachment in the skull bone, osseointegration has been critical in order for the screw to maintain its rigid anchorage in the bone [Tjellström et al., 1981]. Osseointegration is not necessary in order to transmit boneconducted sound. For example, one can use a BAHA on a test rod against the teeth to verify that just a small contact with the teeth is required to get the full sound volume. A similar test was also done in a cadaver study where no difference in cochlear stimulation was found when the transducer casing was applied against aerated cells in the mastoid as compared to when it was firmly attached over the whole same surface by means of bone cement [Håkansson et al., 2010]. Finally, the mandibular joint can efficiently transmit vibrations [Zwislocki, 1953] even if there is no osseointegration involved but instead synovial cavities with an articular disc between the mandibular condyle and the mandibular fossa. It is probably more important that the transmission surface is stiff enough to transmit the relatively small dynamic forces involved without much motion needed.

The new era in bone conduction hearing devices seems to focus on intact skin solutions [Håkansson, 2011]. Some of the new bone conduction hearing devices are dependent on an osseointegrated screw [Baha ${ }^{\circledR}$ Attract (Cochlear Bone Anchored Solutions, Mölnlycke, Sweden), Sophono ${ }^{\circledR}$ (Sophono Inc., Boulder, Colo., USA) and Bonebridge $\left.{ }^{\mathrm{TM}}\right]$. Even if the screws in the Baha Attract, the Sophono and the Bonebridge are not in contact with air and are not subjected to influence from the environment outside the skin, the screw solution can be considered as a weak part of the systems. If the screws get loose, the system will probably not function with the expected efficiency. In the BCI, no screws are used. Instead, the contact with the bone is a flat implant surface where a limited contact with the bone is enough for the effi- 
ciency of the system. It can be argued that no vibrations were applied to the implants in the sheep during the period between the surgeries and that no comparisons can be made with a $\mathrm{BCI}$ positioned in a human. Very little is published in this area but the literature suggests that osseointegration is promoted by vibrations [Ogawa et al., 2014] although no references are found for flat implants to address this issue.

\section{Conclusion}

Histological and histomorphometrical examination of the tissue in close relation to a flat titanium implant surface in sheep skulls, after 8 months of follow-up, indicated ongoing bone remodeling. Moreover, a condensed layer of bone tissue was observed under the implant and was also visualized on CBCT and CT images. In the present study, CBCT and CT have too low a resolution to judge the degree of osseointegration. This study also showed that the condensed bony layer developing over time close to the implant is correlated to a stiffer contact between the implant and the bone and unchanged vibration transmission for cochlear stimulation. A titanium hearing implant with a flat transducer contact with the underlying bone, like the BCI, is a feasible solution for an implantable bone conduction hearing device.

\section{Acknowledgements}

We would like to thank research technician Petra Hammarström Johansson for preparing the cut and ground sections.

The study was partly financed by grants from the Swedish State under the agreement between the Swedish Government and the county councils concerning economic support of research and education of doctors (ALF agreement). The study was also financed by Vinnova (Swedish Innovation Agency), HRF and Promobilia.

\section{Disclosure Statement}

Bo Håkansson holds several patents related to the BCI device. The other authors report no conflicts of interest in this work.

\section{References}

Adibi S, Zhang W, Servos T, O'Neill PN: Cone beam computed tomography in dentistry: what dental educators and learners should know. J Dent Educ 2012;76:1437-1442.

Albrektsson T, Brånemark PI, Hansson HA, Lindström J: Osseointegrated titanium implants. Requirements for ensuring a long-lasting, direct bone-to-implant anchorage in man. Acta Orthop Scand 1981;52:155-170.

Bolind P, Johansson CB, Balshi TJ, Langer B, Albrektsson T: A study of 275 retrieved Brånemark oral implants. Int J Periodontics Restorative Dent 2005;25:425-437.

Brånemark P-I: Tissue Integrated Prosthesis: Osseointegration in Clinical Dentistry. Chicago, Quintessence Publishing, 1985.

Brånemark PI, Hansson BO, Adell R, Breine U, Lindström J, Hallen O, Öhman A: Osseointegrated implants in the treatment of the edentulous jaw. Experience from a 10-year period. Scand J Plastic Reconstr Surg Suppl 1977; 16:1-132.

Buser D, Chappuis V, Kuchler U, Bornstein MM, Wittneben JG, Buser R, Cavusoglu Y, Belser UC: Long-term stability of early implant placement with contour augmentation. J Dent Res 2013;92:176S-182S.

De Vos W, Casselman J, Swennen GR: Cone-beam computerized tomography (CBCT) imaging of the oral and maxillofacial region: a systematic review of the literature. Int J Oral Maxillofac Surg 2009;38:609-625. 
de Wolf MJ, Hol MK, Huygen PL, Mylanus EA, Cremers CW: Clinical outcome of the simplified surgical technique for BAHA implantation. Otol Neurotol 2008;29:1100-1108.

Donath K, Breuner G: A method for the study of undecalcified bones and teeth with attached soft tissues. The SageSchliff (sawing and grinding) technique. J Oral Pathol 1982;11:318-326.

Dun CA, Faber HT, de Wolf MJ, Mylanus EA, Cremers CW, Hol MK: Assessment of more than 1,000 implanted percutaneous bone conduction devices: skin reactions and implant survival. Otol Neurotol 2012;33:192-198.

Eeg-Olofsson M, Håkansson B, Reinfeldt S, Taghavi H, Lund H, Jansson KJ, Håkansson E, Stalfors J: The bone conduction implant-first implantation, surgical and audiologic aspects. Otol Neurotol 2014;35:679-685.

Guldner C, Heinrichs J, Weiss R, Zimmermann AP, Dassinger B, Bien S, Werner JA, Diogo I: Visualisation of the Bonebridge by means of CT and CBCT. Eur J Med Res 2013;18:30.

Håkansson B: The future of bone conduction hearing devices. Adv Otorhinolaryngol 2011;71:140-152.

Håkansson B, Carlsson P, Tjellström A: The mechanical point impedance of the human head, with and without skin penetration. J Acoust Soc Am 1986;80:1065-1075.

Håkansson B, Reinfeldt S, Eeg-Olofsson M, Östli P, Taghavi H, Adler J, Gabrielsson J, Stenfelt S, Granstrom G: A novel bone conduction implant (BCI): engineering aspects and pre-clinical studies. Int J Audiol 2010;49:203-215.

-Hasan I, Dominiak M, Blaszczyszyn A, Bourauel C, Gedrange T, Heinemann F: Radiographic evaluation of bone density around immediately loaded implants. Ann Anat 2014, Epub ahead of print.

Hobson JC, Roper AJ, Andrew R, Rothera MP, Hill P, Green KM: Complications of bone-anchored hearing aid implantation. J Laryngol Otol 2010;124:132-136.

Johansson CB, Morberg P: Importance of ground section thickness for reliable histomorphometrical results. Biomaterials 1995;16:91-95.

Ogawa T, Vandamme K, Zhang X, Naert I, Possemiers T, Chaudhari A, Sasaki K, Duyck J: Stimulation of titanium implant osseointegration through high-frequency vibration loading is enhanced when applied at high acceleration. Calcified Tissue Int 2014;95:467-475.

Rønold HJ, Ellingsen JE: The use of a coin shaped implant for direct in situ measurement of attachment strength for osseointegrating biomaterial surfaces. Biomaterials 2002;23:2201-2209.

Taghavi H, Hakansson B, Eeg-Olofsson M, Johansson CB, Tjellstrom A, Reinfeldt S, Bergqvist T, Olsson J: A vibration investigation of a flat surface contact to skull bone for direct bone conduction transmission in sheep skulls in vivo. Otol Neurotol 2013;34:690-698.

Tjellström A, Lindström J, Hallen 0, Albrektsson T, Brånemark PI: Osseointegrated titanium implants in the temporal bone. A clinical study on bone-anchored hearing aids. Am J Otol 1981;2:304-310.

-Wallberg E, Granstrom G, Tjellstrom A, Stalfors J: Implant survival rate in bone-anchored hearing aid users: longterm results. J Laryngol Otol 2011;125:1131-1135.

Wennerberg A, Albrektsson T: On implant surfaces: a review of current knowledge and opinions. Int J Oral Maxillofac Implants 2010;25:63-74.

Zwislocki J: Acoustical attenuation between the ears. J Acoust Soc Am 1953;25:752-759. 